TAIWANESE JOURNAL OF MATHEMATICS

Vol. 12, No. 6, pp. 1313-1333, September 2008

This paper is available online at http://www.tjm.nsysu.edu.tw/

\title{
COMBINATORIAL STRUCTURES OF PSEUDOMANIFOLDS AND MATROIDS
}

\author{
Chien-Hung Chen, Shyh-Nan Lee and Mau-Hsiang Shih*
}

\begin{abstract}
We prove a multiple combinatorial Stokes' theorem and a multiple Sperner's lemma and formulate their matroid versions. The combinatorial properties of pseudomanifolds with matroid structures are discussed.
\end{abstract}

\section{INTRODUCTION}

The theory of combinatorics of complexes may be traced back to 1928 [16] when Sperner discovered a combinatorial lemma, that is globally called Sperner's lemma, which gave a drastic simplification of proofs of two topological theorems, namely theorems of invariance of domain and invariance of dimension. In 1929, Knaster, Kuratowski and Mazurkiewicz [4] used Sperner's lemma to give a combinatorial proof of Brouwer's fixed-point theorem. In 1967, Scarf [12] used Sperner's lemma to give a constructive proof of Brouwer's fixed-point theorem and in 1974, Kuhn [5] gave a constructive proof of the fundamental theorem of algebra based on the combinatorial Stokes' theorem. In 1973, Shapley [13] generalized Sperner's lemma with balancd structure, and gave a simple proof of Scarf's theorem concerning the nonemptyness of cores of NTU games. On the other hand, in 1945, Tucker [17] proved a combinatorial lemma in the cube which gave a combinatorial proof of Lusternik-Schnirelmann's topological theorem. In 1967, Ky Fan [2] proved a combinatorial theorem which called combinatorial Stokes' theorem, giving a common generalization of Sperner's lemma and Tucker's combinatorial lemma. In 1992, Shih and Lee [14] proved a combinatorial Lefschetz fixed-point formula, put Sperner's lemma into the form of "alternating sum," and showed that Sperner's

Received February 13, 2008.

2000 Mathematics Subject Classification: 05A19, 52B05, 47H10.

Key words and phrases: Multiple combinatorial Stokes' theorem, Multiple Sperner's lemma, Sperner matroid.

This work was supported, in part, by National Science Council, Taipei, R.O.C., under Grant NSC 92-2115-M-033-006.

*The corresponding author is Mau-Hsiang Shih. 
lemma is the case of the Lefchetz number one for any simplical map on a triangulation of a simplex. In 1989, Bapat [1] proved a multiple Sperner's lemma which gave a combinatorial proof of Gale's theorem [3]. In 1993, Shih and Lee [15] obtained a multiple balanced Sperner's lemma which is a common generalization of Shapley's theorem [13] and Bapat's theorem[1]. In 1998, Lee and Shih [6] proved a multiple Stokes' theorem. In 2008, Meunier [10] gave a different approach of Lee and Shih's result. In 1980, Lovasz [9] gave a matroid version of Sperner's lemma and Lee and Shih [7] gave its completion. The purpose of this paper is to give further generalizations of Lee and Shih's results concerning Stokes' theorem on pseudomanifolds [6] and matroids [7, 8].

\section{Definitions and Notations}

An (abstract) complex is a finite collection $\mathcal{K}$ of nonempty finite sets such that

(K1) if $\sigma$ is a member of $\mathcal{K}$ so is every nonempty subset of $\sigma$.

The members of $\mathcal{K}$ are its simplexes. A simplex $\sigma$ of $\mathcal{K}$ is a $d$-simplex of $\mathcal{K}$ if the cardinality $|\sigma|$ of $\sigma$ is $d+1$, and a subset $\tau$ of $\sigma$ is an $r$-face of $\sigma$ if $\tau$ is an $r$-simplex of $\mathcal{K}$. The union of all simplexes of $\mathcal{K}$ is the vertex set $V(\mathcal{K})$ of $\mathcal{K}$.

A $d$-pseudomanifold is a complex $\mathcal{K}$ having the following two properties:

(M1) Every simplex of $\mathcal{K}$ is a face of at least one $d$-simplex of $\mathcal{K}$.

(M2) Every $(d-1)$-simplex of $\mathcal{K}$ is a common face of at most two distinct $d$ simplexes of $\mathcal{K}$.

A boundary $(d-1)$-simplex of $\mathcal{K}$ is a $(d-1)$-simplex of $\mathcal{K}$ that is a face of exactly one $d$-simplex of $\mathcal{K}$. The set of all boundary $(d-1)$-simplexes of $\mathcal{K}$ is denoted by $\partial \mathcal{K}$.

Let $\sigma=\left\{v_{0}, v_{1}, \ldots, v_{d}\right\}$ be a set of $d+1$ elements. Then there are $(d+1)$ ! orderings of the elements of $\sigma$. Two orderings $I=\left(v_{i_{0}}, v_{i_{1}}, \ldots, v_{i_{d}}\right)$ and $J=$ $\left(v_{j_{0}}, v_{j_{1}}, \ldots, v_{j_{d}}\right)$ have the same orientation, denoted by $I \sim J$, if $\left(\begin{array}{l}v_{i_{0}} v_{i_{1}} \ldots v_{i_{d}} \\ v_{j_{0}} v_{j_{1}} \ldots v_{j_{d}}\end{array}\right)$ is an even permutation. If $d>0$, then the $(d+1)$ ! orderings fall into two equivalence classes. Each of these classes is called an orientation of $\sigma$, and if we fix one of them arbitrarily, the other one is called the opposite orientation. The orientation of $\sigma$ determined by the ordering $\left(v_{i_{0}}, v_{i_{1}}, \ldots, v_{i_{d}}\right)$ is denoted by $(+1)\left[v_{i_{0}}, v_{i_{1}}, \ldots, v_{i_{d}}\right]$ and its opposite orientation is denoted by $(-1)\left[v_{i_{0}}, v_{i_{1}}, \ldots, v_{i_{d}}\right]$. If $d=0$, there is only one class $\left[v_{0}\right]$ and hence only one orientation of $\sigma$. We call the two symbols $(+1)\left[v_{0}\right]$ and $(-1)\left[v_{0}\right]$ orientations on the singleton $\left\{v_{0}\right\}$ and they are defined to be opposite orientations on the singleton $\left\{v_{0}\right\}$. 
Given an orientation $\omega=\varepsilon\left[v_{0}, v_{1}, \ldots, v_{d}\right]$ on the set $\sigma=\left\{v_{0}, v_{1}, \ldots, v_{d}\right\}$ where $\varepsilon= \pm 1$ and $d>0$. For each $k=0,1, \ldots, d$, the induced orientation on $\sigma \backslash\left\{v_{k}\right\}$ from $\omega$ is the well defined orientation $(-1)^{k} \varepsilon\left[v_{0}, \ldots, v_{k-1}, v_{k+1}, \ldots, v_{d}\right]$ on $\sigma \backslash\left\{v_{k}\right\}$.

A $d$-pseudomanifold $\mathcal{K}$ is orientable if there is an orientation-valued map $\omega$ on the set of all $d$-simplexes of $\mathcal{K}$ which satisfies the following two conditions:

(C1) For each $d$-simplex $\sigma$ of $\mathcal{K}, \omega(\sigma)$ is an orientation on $\sigma$.

(C2) If $\tau$ is an $(d-1)$-simplex of $\mathcal{K}$ which is a common face of two distinct $d$-simplexes $\sigma$ and $\sigma^{\prime}$ of $\mathcal{K}$, then $\omega(\sigma)$ and $\omega\left(\sigma^{\prime}\right)$ induce opposite orientations on $\tau$.

The pair $(\mathcal{K}, \omega)$ is called a coherently oriented d-pseudomanifold.

Let $m$ be a positive integer and $\mathcal{K}$ be a $d$-pseudomanifold. An $m$-labelling in $\mathcal{K}$ is a map $\varphi$ on $V(\mathcal{K})$ such that for each vertex $v$ of $\mathcal{K}, \varphi(v)$ is an $m$-tuple $\left(\varphi_{1}(v), \ldots, \varphi_{m}(v)\right)$ where $\varphi_{k}(v) \in\{0,1, \ldots, d\}$ for $k=1, \ldots, m$. Given $\sigma \in \mathcal{K}$ and $f: \sigma \rightarrow\{1, \ldots, m\}$, the pair $(\sigma, f)$ is complete or subcomplete if the set $\left\{\varphi_{f(v)}(v) \mid v \in \sigma\right\}$ is $\{0,1, \ldots, d\}$ or $\{0,1, \ldots, d-1\}$ respectively. When $\mathcal{K}$ is oriented, there is an orientation-valued map $\omega$ on the set of all $d$-simplexes of $\mathcal{K}$ so that $(\mathcal{K}, \omega)$ is a coherently oriented $d$-pseudomanifold. Then the pair $(\sigma, f)$ is positively or negatively complete if $\varphi_{f\left(v_{k}\right)}\left(v_{k}\right)=k$ for $k=0,1, \ldots, d$, and $\omega(\sigma)=(+1)\left[v_{0}, v_{1}, \ldots, v_{d}\right]$ or $\omega(\sigma)=(-1)\left[v_{0}, v_{1}, \ldots, v_{d}\right]$, respectively. Let $\tau$ be a $(d-1)$-face of a $d$-simplex $\sigma$ of $\mathcal{K}$ and let $g: \tau \rightarrow\{1, \ldots, m\}$. Then the pair $(\tau, g)$ is positively or negatively subcomplete in $\sigma$ if the induced orientation on $\tau$ from $\omega(\sigma)$ is, respectively, $(+1)\left[v_{0}, v_{1}, \ldots, v_{d-1}\right]$ or $(-1)\left[v_{0}, v_{1}, \ldots, v_{d-1}\right]$ and $\varphi_{g\left(v_{k}\right)}\left(v_{k}\right)=k$ for $k=0,1, \ldots, d-1$. When $\tau \in \partial \mathcal{K}$ such $d$-simplex $\sigma$ is unique, we simply call the pair $(\tau, g)$ positively or negatively subcomplete. We define

$$
\begin{gathered}
\mathcal{K}(\varphi)=\{(\sigma, f) \mid(\sigma, f) \text { is complete }\}, \\
\partial \mathcal{K}(\varphi)=\{(\tau, g) \mid(\tau, g) \text { is subcomplete and } \tau \in \partial \mathcal{K}\},
\end{gathered}
$$

and, when $(\mathcal{K}, \omega)$ is a coherently oriented $d$-pseudomanifold,

$$
\begin{aligned}
\mathcal{K}^{+}(\varphi) & =\{(\sigma, f) \mid(\sigma, f) \text { is positively complete }\}, \\
\mathcal{K}^{-}(\varphi) & =\{(\sigma, f) \mid(\sigma, f) \text { is negatively complete }\}, \\
\partial \mathcal{K}^{+}(\varphi) & =\{(\tau, g) \mid(\tau, g) \text { is positively subcomplete }\}, \\
\partial \mathcal{K}^{-}(\varphi) & =\{(\tau, g) \mid(\tau, g) \text { is negatively subcomplete }\} .
\end{aligned}
$$

We finally define $\mathcal{K}(\varphi)_{*}, \mathcal{K}^{+}(\varphi)_{*}$ and $\mathcal{K}^{-}(\varphi)_{*}$ to be the sets of the pairs $(\sigma, f)$ of $\mathcal{K}(\varphi), \mathcal{K}^{+}(\varphi)$ and $\mathcal{K}^{-}(\varphi)$ such that $f$ is one-to-one and define $\partial \mathcal{K}(\varphi)_{*}, \partial \mathcal{K}^{+}(\varphi)_{*}$ and $\partial \mathcal{K}^{-}(\varphi)_{*}$ to be the sets of the pairs $(\tau, g)$ of $\partial \mathcal{K}(\varphi), \partial \mathcal{K}^{+}(\varphi)$ and $\partial \mathcal{K}^{-}(\varphi)$ such that $g$ is one-to-one, respectively. 


\section{Multiple Combinatorial Stokes' Theorem}

Theorem 1. Let $\varphi$ be an $m$-labelling in a d-pseudomanifold $\mathcal{K}(d>0)$. Then

$$
|\mathcal{K}(\varphi)| \equiv m|\partial \mathcal{K}(\varphi)| \quad(\bmod 2)
$$

and

$$
\left|\mathcal{K}(\varphi)_{*}\right| \equiv(m-d)\left|\partial \mathcal{K}(\varphi)_{*}\right| \quad(\bmod 2) .
$$

Suppose further, $(\mathcal{K}, \omega)$ is a coherently oriented d-pseudomanifold, then

$$
(-1)^{d}\left\{\left|\mathcal{K}^{+}(\varphi)\right|-\left|\mathcal{K}^{-}(\varphi)\right|\right\}=m\left\{\left|\partial \mathcal{K}^{+}(\varphi)\right|-\left|\partial \mathcal{K}^{-}(\varphi)\right|\right\}
$$

and

$$
(-1)^{d}\left\{\left|\mathcal{K}^{+}(\varphi)_{*}\right|-\left|\mathcal{K}^{-}(\varphi)_{*}\right|\right\}=(m-d)\left\{\left|\partial \mathcal{K}^{+}(\varphi)_{*}\right|-\left|\partial \mathcal{K}^{-}(\varphi)_{*}\right|\right\} .
$$

Proof. Let

$$
S=\{(\sigma, f) \mid \sigma \text { is a } d \text {-simplex and } f: \sigma \rightarrow\{1, \ldots, m\}\}
$$

and

$$
T=\{(\tau, g) \mid \tau \text { is a }(d-1) \text {-simplex and } g: \tau \rightarrow\{1, \ldots, m\}\} .
$$

Define an incidence relation $\prec$ from $T$ to $S$ by $(\tau, g) \prec(\sigma, f)$ if and only if

(R1) $(\sigma, f) \in S$ and $(\tau, g) \in T$,

(R2) $(\tau, g)$ is subcomplete,

(R3) $\tau \subset \sigma$ and $g=\left.f\right|_{\tau}$ (the restriction of $f$ to $\tau$ ).

Put

$$
\begin{aligned}
& S_{1}=\{(\sigma, f) \in S \mid(\sigma, f) \text { is complete }\}, \\
& S_{2}=\{(\sigma, f) \in S \mid(\sigma, f) \text { is subcomplete }\}, \\
& S_{3}=\{(\sigma, f) \in S \mid(\sigma, f) \text { is not complete and not subcomplete }\}, \\
& T_{1}=\{(\tau, g) \in T \mid(\tau, g) \text { is subcomplete and } \tau \in \partial \mathcal{K}\}, \\
& T_{2}=\{(\tau, g) \in T \mid(\tau, g) \text { is subcomplete and } \tau \notin \partial \mathcal{K}\}, \\
& T_{3}=\{(\tau, g) \in T \mid(\tau, g) \text { is not subcomplete }\}
\end{aligned}
$$

Then

$$
\left\{S_{1}, S_{2}, S_{3}\right\} \text { is a partition of } S
$$


and

$$
\left\{T_{1}, T_{2}, T_{3}\right\} \text { is a partition of } T \text {. }
$$

Let

$$
S_{t}=\{s \in S \mid t \prec s\} \quad(t \in T)
$$

and

$$
T_{s}=\{t \in T \mid t \prec s\} \quad(s \in S) .
$$

We claim that

$$
\begin{gathered}
\left|S_{t}\right|=m\left(t \in T_{1}\right), \\
\left|S_{t}\right|=2 m\left(t \in T_{2}\right), \\
\left|S_{t}\right|=0\left(t \in T_{3}\right) .
\end{gathered}
$$

To see (3.7), (3.8) and (3.9), let us fix $t=(\tau, g) \in T$, where

$$
\tau=\left\{v_{0}, v_{1}, \ldots, v_{d-1}\right\} .
$$

Case 1. $t=(\tau, g) \in T_{1}$. Then $\tau \in \partial \mathcal{K}$, so that $\tau$ is a face of exactly one $d$-simplex $\sigma$ of $\mathcal{K}$, say

$$
\sigma=\left\{v_{0}, v_{1}, \ldots, v_{d}\right\}
$$

and there are exactly $m$ extensions $f_{1}, \ldots, f_{m}$ of $g$ to the set $\sigma$ into $\{1, \ldots, m\}$, namely,

$$
f_{j}\left(v_{k}\right)=\left\{\begin{array}{cl}
g\left(v_{k}\right) & \text { if } k=0,1, \ldots, d-1 \\
j & \text { if } k=d
\end{array}\right.
$$

for $j=1, \ldots, m$, thus

$$
S_{t}=\left\{\left(\sigma, f_{1}\right), \ldots,\left(\sigma, f_{m}\right)\right\}
$$

and (3.7) follows.

Case 2. $t=(\tau, g) \in T_{2}$. Then $\tau \notin \partial \mathcal{K}$, so that $\tau$ is a face of exactly two distinct $d$-simplexes $\sigma$ and $\sigma^{\prime}$ of $\mathcal{K}$, say

$$
\sigma=\tau \cup\left\{v_{d}\right\} \text { and } \sigma^{\prime}=\tau \cup\left\{v_{d}^{\prime}\right\} .
$$


For each $j=1, \ldots, m$, let $f_{j}$ and $f_{j}^{\prime}$ be the functions on $\sigma$ and $\sigma^{\prime}$ into $\{1, \ldots, m\}$ defined by

$$
\left.f_{j}\right|_{\tau}=\left.f_{j}^{\prime}\right|_{\tau}=g \text { and } f_{j}\left(v_{d}\right)=f_{j}^{\prime}\left(v_{d}^{\prime}\right)=j,
$$

we have

$$
S_{t}=\left\{\left(\sigma, f_{1}\right), \ldots,\left(\sigma, f_{m}\right)\right\} \cup\left\{\left(\sigma^{\prime}, f_{1}^{\prime}\right), \ldots,\left(\sigma^{\prime}, f_{m}^{\prime}\right)\right\}
$$

and (3.8) follows.

Case 3. $t=(\tau, g) \in T_{3}$. Then $(\tau, g)$ is not subcomplete, so that, by (R2), $S_{t}$ $=\emptyset$ and (3.9) follows.

We next claim that

$$
\begin{aligned}
& \left|T_{s}\right|=1 \quad\left(s \in S_{1}\right) \\
& \left|T_{s}\right|=2 \quad\left(s \in S_{2}\right) \\
& \left|T_{s}\right|=0 \quad\left(s \in S_{3}\right)
\end{aligned}
$$

To see (3.15), (3.16) and (3.17), let us fix $s=(\sigma, f) \in S$, where $\sigma$ is given by (3.11). Let

$$
\tau=\sigma \backslash\left\{v_{d}\right\}
$$

and

$$
g=\left.f\right|_{\tau} .
$$

Then, by (K1), (3.10), (3.18) and (3.19),

$$
(\tau, g) \in T \text {. }
$$

Case 1'. $s=(\sigma, f) \in S_{1}$. Then $(\sigma, f)$ is complete, we may assume that

$$
\varphi_{f\left(v_{k}\right)}\left(v_{k}\right)=k \text { for } k=0,1, \ldots, d .
$$

It follows from (3.18), (3.19) and (3.21) that

$$
(\tau, g) \text { is subcomplete, }
$$

so that, by comparing (3.18), (3.19), (3.20) and (3.22) with (R1), (R2) and (R3), we have

$$
T_{s}=\{(\tau, g)\}
$$


and (3.15) follows.

Case 2'. $s=(\sigma, f) \in S_{2}$. Then $(\sigma, f)$ is subcomplete, so that

$$
\left\{\varphi_{f(v)}(v) \mid v \in \sigma\right\}=\{0,1, \ldots, d-1\} .
$$

By (3.11) and (3.24), we may assume that

$$
\varphi_{f\left(v_{k}\right)}\left(v_{k}\right)=k \text { for } k=0,1, \ldots, d-1
$$

and

$$
\varphi_{f\left(v_{d}\right)}\left(v_{d}\right)=i \text { for some } i \in\{0,1, \ldots, d-1\} .
$$

Put

$$
\tau^{\prime}=\sigma \backslash\left\{v_{i}\right\} \text { and } g^{\prime}=\left.f\right|_{\tau^{\prime}} .
$$

We have

$$
\left(\tau^{\prime}, g^{\prime}\right) \text { is subcomplete, }
$$

it follows that

$$
T_{s}=\left\{(\tau, g),\left(\tau^{\prime}, g^{\prime}\right)\right\}
$$

and (3.16) follows.

Case 3'. $s=(\sigma, f) \in S_{3}$. Then $(\sigma, f)$ is not complete and not subcomplete, so that

$$
\{0,1, \ldots, d-1\} \not \subset\left\{\varphi_{f(v)}(v) \mid v \in \sigma\right\}
$$

thus

$$
T_{s}=\emptyset
$$

and (3.17) follows.

Define $\lambda: T \times S \rightarrow\{0,1\}$ by

$$
\lambda(t, s)= \begin{cases}1, & \text { if } t \prec s \\ 0, & \text { otherwise. }\end{cases}
$$


Then, by (3.6), (3.7), (3.8) and (3.9),

$$
\begin{aligned}
\sum_{t \in T} \sum_{s \in S} \lambda(t, s) & =\sum_{t \in T_{1}} \sum_{s \in S} \lambda(t, s)+\sum_{t \in T_{2}} \sum_{s \in S} \lambda(t, s)+\sum_{t \in T_{3}} \sum_{s \in S} \lambda(t, s) \\
& =\sum_{t \in T_{1}}\left|S_{t}\right|+\sum_{t \in T_{2}}\left|S_{t}\right|+\sum_{t \in T_{3}}\left|S_{t}\right| \\
& =m\left|T_{1}\right|+2 m\left|T_{2}\right|+0\left|T_{3}\right|
\end{aligned}
$$

and, by (3.5), (3.15), (3.16) and (3.17),

$$
\begin{aligned}
\sum_{s \in S} \sum_{t \in T} \lambda(t, s) & =\sum_{s \in S_{1}} \sum_{t \in T} \lambda(t, s)+\sum_{s \in S_{2}} \sum_{t \in T} \lambda(t, s)+\sum_{s \in S_{3}} \sum_{t \in T} \lambda(t, s) \\
& =\sum_{s \in S_{1}}\left|T_{s}\right|+\sum_{s \in S_{2}}\left|T_{s}\right|+\sum_{s \in S_{3}}\left|T_{s}\right| \\
& =\left|S_{1}\right|+2\left|S_{2}\right|+0\left|S_{3}\right| .
\end{aligned}
$$

It follows that

$$
m\left|T_{1}\right|+2 m\left|T_{2}\right|=\left|S_{1}\right|+2\left|S_{2}\right| .
$$

As

$$
S_{1}=\mathcal{K}(\varphi) \text { and } T_{1}=\partial \mathcal{K}(\varphi),
$$

from (3.32) and (3.33), (3.1) is proved.

To see (3.2), let the sets, respectively, $S_{*}, S_{1 *}, S_{2 *}, S_{3 *}, T_{*}, T_{1 *}, T_{2 *}$ and $T_{3 *}$ be the sets of the pairs in $S, S_{1}, S_{2}, S_{3}, T, T_{1}, T_{2}$ and $T_{3}$ such that all the functions $f$ or $g$ in the pairs $(\sigma, f)$ or $(\tau, g)$ are one-to-one. As before, we have

$$
\left\{S_{1 *}, S_{2 *}, S_{3 *}\right\} \text { is a partition of } S_{*}
$$

and

$$
\left\{T_{1 *}, T_{2 *}, T_{3 *}\right\} \text { is a partition of } T_{*} \text {. }
$$

Let

$$
S_{t *}=\left\{s \in S_{*} \mid t \prec s\right\} \quad\left(t \in T_{*}\right)
$$

and

$$
T_{s *}=\left\{t \in T_{*} \mid t \prec s\right\} \quad\left(s \in S_{*}\right) .
$$


We claim that, in case $m>d$,

$$
\begin{aligned}
& \left|S_{t *}\right|=m-d \quad\left(t \in T_{1 *}\right) \\
& \left|S_{t *}\right|=2(m-d) \quad\left(t \in T_{2 *}\right) \\
& \left|S_{t *}\right|=0 \quad\left(t \in T_{3 *}\right) \\
& \left|T_{s *}\right|=1 \quad\left(s \in S_{1 *}\right) \\
& \left|T_{s *}\right|=2 \quad\left(s \in S_{2 *}\right) \\
& \left|T_{s *}\right|=0 \quad\left(s \in S_{3 *}\right) .
\end{aligned}
$$

To see (3.36), (3.37) and (3.38), let us fix $t=(\tau, g) \in T_{*}$. Since $g$ is one-to-one, the cardinality of the image of $\tau$ under $g$ is $|g(\tau)|=d$. If $t=(\tau, g) \in T_{1 *}$, then there are exactly $m-d$ injective extentions of $g$ to $\sigma$ into $\{1, \ldots, m\}$, namely,

$$
S_{t *}=\left\{\left(\sigma, f_{j}\right) \mid j \in\{1, \ldots, m\} \backslash g(\tau)\right\}\left(t \in T_{1 *}\right)
$$

where $\tau, \sigma$ and $f_{j}$ are the same as in (3.10), (3.11) and (3.12) respectively. Similarly, if we define $f_{j}$ and $f_{j}^{\prime}$ as in (3.14), then we have

$$
\begin{aligned}
S_{t *}= & \left\{\left(\sigma, f_{j}\right) \mid j \in\{1, \ldots, m\} \backslash g(\tau)\right\} \\
& \cup\left\{\left(\sigma^{\prime}, f_{j}^{\prime}\right) \mid j \in\{1, \ldots, m\} \backslash g(\tau)\right\} \quad\left(t \in T_{2 *}\right) .
\end{aligned}
$$

It is clear that

$$
S_{t *}=\emptyset\left(t \in T_{3 *}\right) .
$$

This proves (3.36), (3.37) and (3.38). The same argument in the proof of (3.15), (3.16) and (3.17) shows that (3.39), (3.40) and (3.41) are true. It follows from (3.34) (3.41) that

$$
\sum_{t \in T_{*}} \sum_{s \in S_{*}} \lambda(t, s)=(m-d)\left|T_{1 *}\right|+2(m-d)\left|T_{2 *}\right|
$$

and

$$
\sum_{s \in S_{*}} \sum_{t \in T_{*}} \lambda(t, s)=\left|S_{1 *}\right|+2\left|S_{2 *}\right|,
$$


so that

$$
(m-d)\left|T_{1 *}\right|+2(m-d)\left|T_{2 *}\right|=\left|S_{1 *}\right|+2\left|S_{2 *}\right| .
$$

As

$$
S_{1 *}=\mathcal{K}(\varphi)_{*} \text { and } T_{1 *}=\partial \mathcal{K}(\varphi)_{*},
$$

from (3.45) and (3.46), (3.2) is proved.

Suppose further, $(\mathcal{K}, \omega)$ is a coherently oriented $d$-pseudomanifold. Put

$$
\begin{aligned}
& S_{t}^{+}=\left\{(\sigma, f) \in S_{t} \mid(\tau, g) \text { is positively subcomplete in } \sigma\right\} \quad(t=(\tau, g) \in T), \\
& S_{t}^{-}=\left\{(\sigma, f) \in S_{t} \mid(\tau, g) \text { is negatively subcomplete in } \sigma\right\} \quad(t=(\tau, g) \in T), \\
& T_{s}^{+}=\left\{(\tau, g) \in T_{s} \mid(\tau, g) \text { is positively subcomplete in } \sigma\right\} \quad(s=(\sigma, f) \in S), \\
& T_{s}^{-}=\left\{(\tau, g) \in T_{s} \mid(\tau, g) \text { is negatively subcomplete in } \sigma\right\} \quad(s=(\sigma, f) \in S) .
\end{aligned}
$$

Note that

$$
\left\{\mathcal{K}^{+}(\varphi), \mathcal{K}^{-}(\varphi)\right\} \text { partitions } S_{1}
$$

and

$$
\left\{\partial \mathcal{K}^{+}(\varphi), \partial \mathcal{K}^{-}(\varphi)\right\} \text { partitions } T_{1}
$$

We claim that

$$
\begin{array}{cc}
\left|S_{t}^{+}\right|=m \text { and }\left|S_{t}^{-}\right|=0 \quad\left(t \in \partial \mathcal{K}^{+}(\varphi)\right), \\
\left|S_{t}^{+}\right|=0 \text { and }\left|S_{t}^{-}\right|=m \quad\left(t \in \partial \mathcal{K}^{-}(\varphi)\right), \\
\left|S_{t}^{+}\right|=\left|S_{t}^{-}\right|=m \quad\left(t \in T_{2}\right), \\
\left|S_{t}^{+}\right|=\left|S_{t}^{-}\right|=0 \quad\left(t \in T_{3}\right) .
\end{array}
$$

If $t=(\tau, g) \in \partial \mathcal{K}^{+}(\varphi)$, then $(\tau, g)$ is positively complete, so that

$$
S_{t}^{+}=\left\{\left(\sigma, f_{1}\right), \ldots,\left(\sigma, f_{m}\right)\right\} \text { and } S_{t}^{-}=\emptyset,
$$

where $\tau, \sigma$ and $f_{j}$ are given by (3.10), (3.11) and (3.12), this proves (3.49). Similarly, if $t=(\tau, g) \in \partial \mathcal{K}^{-}(\varphi)$ then $S_{t}^{+}=\emptyset$ and $S_{t}^{-}=\left\{\left(\sigma, f_{1}\right), \ldots,\left(\sigma, f_{m}\right)\right\}$ and (3.50) follows. It follows from (M2), (C2) that if $t \in T_{2}$ then $S_{t}^{+}$is one of the two sets $\left\{\left(\sigma, f_{1}\right), \ldots,\left(\sigma, f_{m}\right)\right\}$ and $\left\{\left(\sigma^{\prime}, f_{1}^{\prime}\right), \ldots,\left(\sigma^{\prime}, f_{m}^{\prime}\right)\right\}$ and $S_{t}^{-}$is the other, where 
$\sigma, \sigma^{\prime}, f_{j}$ and $f_{j}^{\prime}$ are given by (3.13) and (3.14), this proves (3.51). If $t=(\tau, g) \in T_{3}$ then $(\tau, g)$ is not subcomplete, so that $S_{t}^{+}=S_{t}^{-}=\emptyset$ and (3.52) follows.

We next claim that

$$
\begin{gathered}
\left|T_{s}^{+}\right|=\frac{1+(-1)^{d}}{2} \text { and }\left|T_{s}^{-}\right|=\frac{1-(-1)^{d}}{2} \quad\left(s \in \mathcal{K}^{+}(\varphi)\right), \\
\left|T_{s}^{+}\right|=\frac{1-(-1)^{d}}{2} \text { and }\left|T_{s}^{-}\right|=\frac{1+(-1)^{d}}{2} \quad\left(s \in \mathcal{K}^{-}(\varphi)\right), \\
\left|T_{s}^{+}\right|=\left|T_{s}^{-}\right|=1 \quad\left(s \in S_{2}\right), \\
\left|T_{s}^{+}\right|=\left|T_{s}^{-}\right|=0 \quad\left(s \in S_{3}\right) .
\end{gathered}
$$

Let $s=(\sigma, f)$ and $\omega(\sigma)=\varepsilon\left[v_{0}, v_{1}, \ldots, v_{d}\right](\varepsilon= \pm 1)$. If $s=(\sigma, f) \in \mathcal{K}^{+}(\varphi)$, then $\omega(\sigma)=(+1)\left[v_{0}, v_{1}, \ldots, v_{d}\right]$ with the assumption (3.21), so that (3.22) holds and $\omega(\sigma)$ induces $(-1)^{d}\left[v_{0}, v_{1}, \ldots, v_{d-1}\right]$ on $\tau$, thus

$$
\begin{array}{ll}
T_{s}^{+}=\{(\tau, g)\} \text { and } T_{s}^{-}=\emptyset & \text { if } d \text { is even, } \\
T_{s}^{+}=\emptyset \text { and } T_{s}^{-}=\{(\tau, g)\} & \text { if } d \text { is odd, }
\end{array}
$$

where $\tau$ and $g$ are given in (3.18) and (3.19) respectively. This proves (3.53). Similarly, if $s=(\sigma, f) \in \mathcal{K}^{-}(\varphi)$, then

$$
\begin{array}{ll}
T_{s}^{+}=\emptyset \text { and } T_{s}^{-}=\{(\tau, g)\} & \text { if } d \text { is even, } \\
T_{s}^{+}=\{(\tau, g)\} \text { and } T_{s}^{-}=\emptyset & \text { if } d \text { is odd, }
\end{array}
$$

and (3.54) follows. Next, if $s=(\sigma, f) \in S_{2}$, then by (3.18) and (3.27) we have

$$
\begin{aligned}
& \omega(\sigma) \text { induces }(-1)^{d} \varepsilon\left[v_{0}, v_{1}, \ldots, v_{d-1}\right] \text { on } \tau, \\
& \omega(\sigma) \text { induces }(-1)^{d+1} \varepsilon\left[v_{0}, v_{1}, \ldots, v_{i-1}, v_{d}, v_{i+1}, \ldots, v_{d-1}\right] \text { on } \tau^{\prime},
\end{aligned}
$$

so that by (3.25), (3.26) and (3.27), one of the two pairs $(\tau, g)$ and $\left(\tau^{\prime}, g^{\prime}\right)$ is positively subcomplete in $\sigma$ and the other one is negatively subcomplete in $\sigma$, thus (3.55) is true. Finally, if $s=(\sigma, f) \in S_{3}$, then (3.17) implies that $\left|T_{s}^{+}\right|=\left|T_{s}^{-}\right|=0$, so that (3.56) is true.

Define $\Lambda: T \times S \rightarrow\{-1,0,1\}$ by

$$
\Lambda(t, s)=\left\{\begin{aligned}
1, & \text { if } t \prec s \text { and }(\tau, g) \text { is positively subcomplete in } \sigma \\
-1, & \text { if } t \prec s \text { and }(\tau, g) \text { is negatively subcomplete in } \sigma \\
0, & \text { otherwise. }
\end{aligned}\right.
$$


where $t=(\tau, g) \in T$ and $s=(\sigma, f) \in S$. Then by (3.48) (3.52),

$$
\begin{aligned}
\sum_{t \in T} \sum_{s \in S} \Lambda(t, s) & =\sum_{t \in T}\left(\left|S_{t}^{+}\right|-\left|S_{t}^{-}\right|\right) \\
& =\left(\sum_{t \in \partial \mathcal{K}^{+}(\varphi)}+\sum_{t \in \partial \mathcal{K}^{-}(\varphi)}+\sum_{t \in T_{2}}+\sum_{t \in T_{3}}\right)\left(\left|S_{t}^{+}\right|-\left|S_{t}^{-}\right|\right) \\
& =\left|\partial \mathcal{K}^{+}(\varphi)\right|(m-0)+\left|\partial \mathcal{K}^{-}(\varphi)\right|(0-m)+\left|T_{2}\right|(m-m)+\left|T_{3}\right|(0-0) \\
& =m\left\{\left|\partial \mathcal{K}^{+}(\varphi)\right|-\left|\partial \mathcal{K}^{-}(\varphi)\right|\right\}
\end{aligned}
$$

and, by (3.47) and (3.53) (3.56),

$$
\begin{aligned}
\sum_{s \in S} \sum_{t \in T} \Lambda(t, s)= & \sum_{s \in S}\left(\left|T_{s}^{+}\right|-\left|T_{s}^{-}\right|\right) \\
= & \left(\sum_{s \in \mathcal{K}^{+}(\varphi)}+\sum_{s \in \mathcal{K}^{-}(\varphi)}+\sum_{s \in S_{2}}+\sum_{s \in S_{3}}\right)\left(\left|T_{s}^{+}\right|-\left|T_{s}^{-}\right|\right) \\
= & \left|\mathcal{K}^{+}(\varphi)\right|\left\{\frac{1+(-1)^{d}}{2}-\frac{1-(-1)^{d}}{2}\right\}+ \\
& \left|\mathcal{K}^{-}(\varphi)\right|\left\{\frac{1-(-1)^{d}}{2}-\frac{1+(-1)^{d}}{2}\right\}+\left|S_{2}\right|(1-1)+\left|S_{3}\right|(0-0) \\
= & (-1)^{d}\left\{\left|\mathcal{K}^{+}(\varphi)\right|-\left|\mathcal{K}^{-}(\varphi)\right|\right\},
\end{aligned}
$$

thus (3.3) holds. If $m>d$, by a similar argument as in the proof of (3.2) and (3.3), the equality (3.4) holds. We mention that if $m \leq d$, then both sides of (3.2) and (3.4) are zeros, thus $(3.1) \sim(3.4)$ hold for any positive integers $m$ and $d$. This completes the proof of Theorem 1 .

\section{Multiple Sperner's Lemma}

A subset $\sigma=\left\{v_{0}, v_{1}, \ldots, v_{d}\right\}$ of a Euclidean space is affinely independent if

(A1) $\sum_{k=0}^{d} \lambda_{k} v_{k}=0$ and $\sum_{k=0}^{d} \lambda_{k}=0$ imply each $\lambda_{k}=0$,

the convex hull conv $\sigma$ of the affinely independent set $\sigma$ is called a (geometric) $d$-simplex with the vertices $v_{0}, v_{1}, \ldots, v_{d}$, sometimes we denote this simplex by $\overline{v_{0} v_{1} \ldots v_{d}}$, thus

$$
\overline{v_{0} v_{1} \cdots v_{d}}=\left\{\sum_{k=0}^{d} \lambda_{k} v_{k} \mid \sum_{k=0}^{d} \lambda_{k}=1, \text { each } \lambda_{k} \geq 0\right\},
$$


for $0 \leq r \leq d$ and $0 \leq k_{0}<k_{1}<\ldots<k_{r} \leq d$, the simplex $\overline{v_{k_{0}} v_{k_{1}} \ldots v_{k_{r}}}$ is called an $r$-face of $\overline{v_{0} v_{1} \ldots v_{d}}$.

A finite collection $T$ of (geometric) simplexes is called a triangulation of a $d$-simplex $\overline{a_{0} a_{1} \ldots a_{d}}$ if it satisfies the following three conditions:

(T1) $\overline{a_{0} a_{1} \ldots a_{d}}=\bigcup_{s \in T} s$.

(T2) If $s \in T$ and $t$ is a face of $s$ then $t \in T$.

(T3) If $s, t \in T$ and $s \cap t \neq \emptyset$, then $s \cap t$ is a common face of $s$ and $t$.

A point $v \in \overline{a_{0} a_{1} \ldots a_{d}}$ is a vertex of $T$ if $v$ is a vertex of some simplex of $T$. The set of all vertices of $T$ is denoted by $V(T)$. The collection $\widetilde{T}$ of all subsets $\left\{v_{0}, v_{1}, \ldots, v_{r}\right\}$ of $V(T)$ such that $\overline{v_{0} v_{1} \ldots v_{r}} \in T$ is the vertex scheme of $T$ and which is a $d$-pseudomanifold. For each $d$-simplex $\sigma=\left\{v_{0}, v_{1}, \ldots, v_{d}\right\}$ of $\widetilde{T}$, the canonical orientation $\omega(\sigma)$ on $\sigma$ is $(+1)\left[v_{0}, v_{1}, \ldots, v_{d}\right]$ or $(-1)\left[v_{0}, v_{1}, \ldots, v_{d}\right]$ according as $\operatorname{det}\left(\lambda_{i j}\right)>0$ or $\operatorname{det}\left(\lambda_{i j}\right)<0$ respectively, where $\left(\lambda_{i j}\right)$ is the $d+1$ square matrix satisfying

$$
v_{i}=\sum_{j=0}^{d} \lambda_{i j} a_{j} \quad\left(\sum_{j=0}^{d} \lambda_{i j}=1\right) \quad \text { for } i=0,1, \ldots, d .
$$

Then $(\widetilde{T}, \omega)$ becomes a coherently oriented $d$-pseudomanifold.

An $m$-labelling $\varphi$ in $\widetilde{T}$ is Sperner if it satisfies the following facial condition:

(F1) For each $v \in V(T)$ and each $j \in\{1, \ldots, m\}$,

$$
\begin{aligned}
& \qquad v \in \overline{a_{k_{0}} a_{k_{1}} \ldots a_{k_{r}}} \quad \text { implies } \quad \varphi_{j}(v) \in\left\{k_{0}, k_{1}, \ldots, k_{r}\right\} \\
& \text { whenever } 0 \leq r \leq d \text { and } 0 \leq k_{0}<k_{1}<\ldots<k_{r} \leq d .
\end{aligned}
$$

Theorem 2. Let $\varphi$ be an m-labelling in the vertex scheme $\widetilde{T}$ of a triangulation $T$ of a given $d$-simplex $\overline{a_{0} a_{1} \ldots a_{d}}$. If $\varphi$ is Sperner, then, with the canonical orientation $\omega$, we have

$$
\left|\widetilde{T}^{+}(\varphi)\right|-\left|\widetilde{T}^{-}(\varphi)\right|=m^{d+1},
$$

and if $m>d$, we have

$$
\left|\widetilde{T}^{+}(\varphi)_{*}\right|-\left|\widetilde{T}^{-}(\varphi)_{*}\right|=m(m-1) \ldots(m-d) .
$$

Proof. For each $k=0,1, \ldots, d$, let $T_{k}$ be the restricted triangulation of $T$ to the $k$-simplex $\overline{a_{0} a_{1} \ldots a_{k}}$, that is,

$$
T_{k}=\left\{s \in T \mid s \subset \overline{a_{0} a_{1} \ldots a_{k}}\right\} .
$$


Then $\left(\widetilde{T}_{k}, \omega_{k}\right)$ is a coherently oriented $k$-pseudomanifold where $\omega_{k}$ is the canonical orientation on the set of all $k$-simplexes of $\widetilde{T}_{k}$. Precisely,

$$
\omega_{k}\left(\left\{v_{0}, v_{1}, \ldots, v_{k}\right\}\right)=\varepsilon\left[v_{0}, v_{1}, \ldots, v_{k}\right] \quad(\varepsilon= \pm 1)
$$

if and only if

$$
\operatorname{det}\left(\lambda_{i j}\right)_{(k+1) \times(k+1)}=\varepsilon\left|\operatorname{det}\left(\lambda_{i j}\right)_{(k+1) \times(k+1)}\right|
$$

where

$$
v_{i}=\sum_{j=0}^{k} \lambda_{i j} a_{j} \quad\left(\sum_{j=0}^{k} \lambda_{i j}=1\right) \quad \text { for } i=0,1, \ldots, k .
$$

It follows from (F1) that the restriction of $\varphi$ to $V\left(T_{k}\right)$ is a Sperner $m$-labelling in $\widetilde{T}_{k}$. We shall show that

$$
\begin{gathered}
\widetilde{T}_{0}^{+}(\varphi)|=m, \quad| \widetilde{T}_{0}^{-}(\varphi) \mid=0, \\
\left|\partial \widetilde{T}_{k}^{+}(\varphi)\right|-\left|\partial \widetilde{T}_{k}^{-}(\varphi)\right|=(-1)^{k}\left\{\left|\widetilde{T}_{k-1}^{+}(\varphi)\right|-\left|\widetilde{T}_{k-1}^{-}(\varphi)\right|\right\}, \\
\left|\widetilde{T}_{0}^{+}(\varphi)_{*}\right|=m, \quad\left|\widetilde{T}_{0}^{-}(\varphi)_{*}\right|=0, \\
\left|\partial \widetilde{T}_{k}^{+}(\varphi)_{*}\right|-\left|\partial \widetilde{T}_{k}^{-}(\varphi)_{*}\right|=(-1)^{k}\left\{\left|\widetilde{T}_{k-1}^{+}(\varphi)_{*}\right|-\left|\widetilde{T}_{k-1}^{-}(\varphi)_{*}\right|\right\},
\end{gathered}
$$

where $0<k \leq d$.

Observe that Theorem 1, (4.8) and (4.10) will imply

$$
\left|\widetilde{T}_{k}^{+}(\varphi)\right|-\left|\widetilde{T}_{k}^{-}(\varphi)\right|=m\left\{\left|\widetilde{T}_{k-1}^{+}(\varphi)\right|-\left|\widetilde{T}_{k-1}^{-}(\varphi)\right|\right\}
$$

and

$$
\left|\widetilde{T}_{k}^{+}(\varphi)_{*}\right|-\left|\widetilde{T}_{k}^{-}(\varphi)_{*}\right|=(m-k)\left\{\left|\widetilde{T}_{k-1}^{+}(\varphi)_{*}\right|-\left|\widetilde{T}_{k-1}^{-}(\varphi)_{*}\right|\right\},
$$

so that (4.1) will follow from (4.7) and (4.11) and (4.2) will follow from (4.9) and (4.12).

By (4.3), $T_{0}=\left\{\overline{a_{0}}\right\}$, so that, by (F1),

$$
\varphi\left(a_{0}\right)=\left(\varphi_{1}\left(a_{0}\right), \ldots, \varphi_{m}\left(a_{0}\right)\right)=(0, \ldots, 0)
$$

it follows from (4.4), (4.5), (4.6) and (4.13) that

$$
\widetilde{T}_{0}^{+}(\varphi)=\left\{\left(\left\{a_{0}\right\}, f_{j}\right) \mid j=1, \ldots, m\right\} \text { and } \widetilde{T}_{0}^{-}(\varphi)=\emptyset
$$


where $f_{j}\left(a_{0}\right)=j$ for $j=1, \ldots, m$, thus (4.7) is true.

As $\left\{a_{0}\right\}$ is a singleton, each $f_{j}$ in (4.14) is one-to-one, so that

$$
\widetilde{T}_{0}^{+}(\varphi)_{*}=\widetilde{T}_{0}^{+}(\varphi) \text { and } \widetilde{T}_{0}^{-}(\varphi)_{*}=\widetilde{T}_{0}^{-}(\varphi),
$$

thus (4.9) is also true.

To see (4.8) and (4.10), let $g: \tau \rightarrow\{1, \ldots, m\}$ where

$$
\tau=\left\{v_{0}, v_{1}, \ldots, v_{k-1}\right\}
$$

and

$$
\varphi_{g\left(v_{j}\right)}\left(v_{j}\right)=j \text { for } j=0,1, \ldots, k-1 .
$$

Then (4.3) and (F1) imply the following (4.18) and (4.19) are equivalent:

$$
\begin{gathered}
(\tau, g) \in \partial \widetilde{T}_{k}(\varphi), \\
(\tau, g) \in \widetilde{T}_{k-1}(\varphi) .
\end{gathered}
$$

We claim that

$$
\begin{aligned}
\partial \widetilde{T}_{k}^{ \pm}(\varphi) & =\widetilde{T}_{k-1}^{ \pm}(\varphi) \text { if } k \text { is even } \\
\partial \widetilde{T}_{k}^{ \pm}(\varphi) & =\widetilde{T}_{k-1}^{\mp}(\varphi) \text { if } k \text { is odd }
\end{aligned}
$$

and

$$
\partial \widetilde{T}_{k}^{ \pm}(\varphi)_{*}=\widetilde{T}_{k-1}^{ \pm}(\varphi)_{*} \text { if } k \text { is even }
$$

$$
\partial \widetilde{T}_{k}^{ \pm}(\varphi)_{*}=\widetilde{T}_{k-1}^{\mp}(\varphi)_{*} \text { if } k \text { is odd. }
$$

It is clear that (4.8) will follow from (4.20) and (4.21) and (4.10) will follow from (4.22) and (4.23).

Now suppose (4.18) and (4.19) hold, let

$$
\sigma=\left\{v_{0}, v_{1}, \ldots, v_{k}\right\} \in \widetilde{T}_{k} .
$$

Then $\tau$ is a face of $\sigma$, by (4.18), $\tau$ is a boundary $(k-1)$-simplex of $\widetilde{T}_{k}$, so that such a $k$-simplex $\sigma$ is unique. By (4.3) and (4.24), we may assume that (4.6) holds, and by (4.16), (4.19) and the affine independence of $\left\{a_{0}, a_{1}, \ldots, a_{k}\right\}$, we have

$$
\lambda_{i k}=0 \text { for } i=0,1, \ldots, k-1
$$


and

$$
\lambda_{k k}>0,
$$

so that, by (4.25),

$$
\operatorname{det}\left(\lambda_{i j}\right)_{(k+1) \times(k+1)}=\lambda_{k k} \operatorname{det}\left(\lambda_{i j}\right)_{k \times k} .
$$

Let

$$
\omega_{k}(\sigma)=\varepsilon\left[v_{0}, v_{1}, \ldots, v_{k}\right]
$$

Then $\omega_{k}(\sigma)$ induces

$$
(-1)^{k} \varepsilon\left[v_{0}, v_{1}, \ldots, v_{k-1}\right]
$$

on $\tau$. By (4.4), (4.5), (4.6), (4.26), (4.27) and (4.28), we have

$$
\operatorname{det}\left(\lambda_{i j}\right)_{k \times k}=\varepsilon\left|\operatorname{det}\left(\lambda_{i j}\right)_{k \times k}\right|
$$

so that, by replacing $k$ by $k-1$ in (4.4), (4.5) and (4.6), we have

$$
\omega_{k-1}(\tau)=\varepsilon\left[v_{0}, v_{1}, \ldots, v_{k-1}\right] .
$$

It follows from (4.17), (4.29) and (4.31) that $(4.20) \sim(4.23)$ hold. This completes the proof.

\section{Combinatorial Formulae and Matroids}

An ordered pair $(E, \mathcal{I})$ is a matroid if $E$ is a finite set and $\mathcal{I}$ is a collection of subsets of $E$ such that the following three conditions are satisfied:

(I1) $\emptyset \in \mathcal{I}$.

(I2) If $I \in \mathcal{I}$ and $I^{\prime} \subset I$, then $I^{\prime} \in \mathcal{I}$.

(I3) If $I_{1} \in \mathcal{I}$ and $I_{2} \in \mathcal{I}$ with $\left|I_{1}\right|<\left|I_{2}\right|$, then $I_{1} \cup\{e\} \in \mathcal{I}$ for some $e \in I_{2} \backslash I_{1}$.

In the matroid $(E, \mathcal{I})$, a subset of $E$ is independent if it is a member of $\mathcal{I}$ and dependent if it is not independent, a maximal independent subset of $E$ is a basis, a minimal dependent subset of $E$ is a circuit, $e \in E$ is a loop if the singleton $\{e\}$ is a circuit, the number

$$
r(X)=\max \{|I| \mid I \subset X, I \in \mathcal{I}\}
$$

is the rank of $X$, where $X \subset E$, and the closure (or span) of $X$ is the set

$$
c l(X)=\{e \in E \mid r(X \cup\{e\})=r(X)\} .
$$


The number $r(E)$ is called the rank of the matroid $(E, \mathcal{I})$. It is well known that all bases are equicardinal. For all necessary background materials we refer to Oxley [11].

Let $B=\left(b_{0}, b_{1}, \ldots, b_{d}\right)$ be an ordered basis of a matroid $(E, \mathcal{I})$ of rank $d+1$ and

$$
F_{j}=c l\left(\left\{b_{0}, b_{1}, \ldots, b_{j}\right\}\right) \text { for } j=0,1, \ldots, d \text {. }
$$

An ordering $\left(e_{0}, e_{1}, \ldots, e_{k}\right)$ of $k+1(0 \leq k \leq d)$ elements of $E$ is a $B$-sequence if

$$
e_{0} \in F_{0} \text { and } e_{j} \in F_{j} \backslash F_{j-1} \text { for } j=1, \ldots, k .
$$

Let $\psi_{B}: E \rightarrow\{0,1, \ldots, d\}$ be the function defined by

$$
\begin{gathered}
\psi_{B}(e)=0 \text { if } e \in F_{0} \\
\psi_{B}(e)=j \text { if } e \in F_{j} \backslash F_{j-1} \text { for } j=1, \ldots, d .
\end{gathered}
$$

Then we have the following properties:

(B1) $\operatorname{cl}(\emptyset) \subset F_{0} \subset F_{1} \subset \ldots \subset F_{d}=E(\operatorname{cl}(\emptyset)$ is the set of all loops in $E)$,

(B2) if $\left(e_{0}, e_{1}, \ldots, e_{k}\right)$ is a $B$-sequence, then the rank $r\left(\left\{e_{0}, e_{1}, \ldots, e_{k}\right\}\right)$ is $k$ or $k+1$ provided $e_{0}$ is a loop or not,

(B3) $\left(e_{0}, e_{1}, \ldots, e_{k}\right)$ is a $B$-sequence if and only if

$$
\psi_{B}\left(e_{j}\right)=j \quad \text { for } \quad j=0,1, \ldots, k .
$$

Let $\mathcal{K}$ be a $d$-pseudomanifold, $B=\left(b_{0}, b_{1}, \ldots, b_{d}\right)$ an ordered basis of a matroid $(E, \mathcal{I})$ of rank $d+1$. If $\phi$ is a map from $V(\mathcal{K})$ into $E^{m}$, the Cartesian product $E \times \cdots \times E$ of $m$ factors, we shall write

$$
\phi(v)=\left(\phi_{1}(v), \ldots, \phi_{m}(v)\right)
$$

and

$$
\left(\psi_{B} \circ \phi\right)(v)=\left(\left(\psi_{B} \circ \phi_{1}\right)(v), \ldots,\left(\psi_{B} \circ \phi_{m}\right)(v)\right)
$$

for $v \in V(\mathcal{K})$, where $\psi_{B}$ is given by (5.3) and (5.4), so that $\varphi=\psi_{B} \circ \phi$ is an $m$ labelling in $\mathcal{K}$ and we may consider a given pair $(\sigma, f)$ which is complete, subcomplete or not by using the map $\phi$ and the ordered basis $B$ instead of the $m$-labelling $\varphi$. We may consider the orientations or the induced orientations when $(\mathcal{K}, \omega)$ is coherently oriented. We call a pair $(\sigma, f) B$-complete, positively B-complete or 
negatively $B$-complete, (relative to $\phi$ and $B$ ) if it is complete, positively complete or negatively complete (relative to $\varphi$ ) and a pair $(\tau, g) B$-subcomplete, positively $B$-subcomplete or negatively $B$-subcomplete if it is subcomplete, positively subcomplete or negatively subcomplete, and we write $\mathcal{K}_{B}(\phi), \mathcal{K}_{B}^{+}(\phi), \mathcal{K}_{B}^{-}(\phi), \partial \mathcal{K}_{B}(\phi)$, $\partial \mathcal{K}_{B}^{+}(\phi)$, and $\partial \mathcal{K}_{B}^{-}(\phi)$, in place of $\mathcal{K}(\varphi), \mathcal{K}^{+}(\varphi), \mathcal{K}^{-}(\varphi), \partial \mathcal{K}(\varphi), \partial \mathcal{K}^{+}(\varphi)$, and $\partial \mathcal{K}^{-}(\varphi)$ respectively. The notations such as $\mathcal{K}_{B}(\phi)_{*}, \ldots, \partial \mathcal{K}_{B}^{-}(\phi)_{*}$ are defined by a similar way.

The following Theorem 3 is a direct consequence of Theorem 1.

Theorem 3. Let $\phi: V(\mathcal{K}) \rightarrow E^{m}$ where $\mathcal{K}$ is a d-pseudomanifold $(d>0)$ and $(E, \mathcal{I})$ is a matroid of rank $d+1$. Then for each ordered basis $B$ of $(E, \mathcal{I})$,

$$
\left|\mathcal{K}_{B}(\phi)\right| \equiv m\left|\partial \mathcal{K}_{B}(\phi)\right| \quad(\bmod 2)
$$

and

$$
\left|\mathcal{K}_{B}(\phi)_{*}\right| \equiv(m-d)\left|\partial \mathcal{K}_{B}(\phi)_{*}\right| \quad(\bmod 2) .
$$

Suppose further, $(\mathcal{K}, \omega)$ is a coherently oriented d-pseudomanifold, then

$$
(-1)^{d}\left\{\left|\mathcal{K}_{B}^{+}(\phi)\right|-\left|\mathcal{K}_{B}^{-}(\phi)\right|\right\}=m\left\{\left|\partial \mathcal{K}_{B}^{+}(\phi)\right|-\left|\partial \mathcal{K}_{B}^{-}(\phi)\right|\right\}
$$

and

$$
(-1)^{d}\left\{\left|\mathcal{K}_{B}^{+}(\phi)_{*}\right|-\left|\mathcal{K}_{B}^{-}(\phi)_{*}\right|\right\}=(m-d)\left\{\left|\partial \mathcal{K}_{B}^{+}(\phi)_{*}\right|-\left|\partial \mathcal{K}_{B}^{-}(\phi)_{*}\right|\right\} .
$$

The following Theorem 4 is corresponding to Theorem 2 .

Theorem 4. Let $\phi: V(\widetilde{T}) \rightarrow E^{m}$ where $\widetilde{T}$ is the vertex scheme of a triangulation $T$ of a d-simplex $\overline{a_{0} a_{1} \ldots a_{d}}$ and $(E, \mathcal{I})$ is a matroid of rank $d+1$ with an ordered basis $B$. If $\phi$ satisfies the facial condition (relative to the ordered basis $B)$ :

for each $v \in V(\widetilde{T})$ and each $j \in\{1, \ldots, m\}$,

$$
v \in \overline{a_{k_{0}} a_{k_{1}} \ldots a_{k_{r}}} \quad \text { implies } \quad\left(\psi_{B} \circ \phi_{j}\right)(v) \in\left\{k_{0}, k_{1}, \ldots, k_{r}\right\}
$$

whenever $0 \leq r \leq d$ and $0 \leq k_{0}<k_{1} \ldots<k_{r} \leq d$, then, with the canonical orientation $\omega$, we have

$$
\left|\widetilde{T}_{B}^{+}(\phi)\right|-\left|\widetilde{T}_{B}^{-}(\phi)\right|=m^{d+1}
$$

and

$$
\left|\widetilde{T}_{B}^{+}(\phi)_{*}\right|-\left|\widetilde{T}_{B}^{-}(\phi)_{*}\right|=m(m-1) \ldots(m-d) .
$$


Proof. By the facial condition (relative to $B$ ), the $m$-labelling $\varphi=\psi_{B} \circ \phi$ is Sperner. Thus the theorem follows from Theorem 2.

Sometimes we are interested in the combinatorics of a pseudomanifold with an independence structure. This is indeed a special case of Theorem 3 with $m=$ $1, E=V(\mathcal{K})$ and $\phi=i d_{V(\mathcal{K})}$ the identity map on $V(\mathcal{K})$. Precisely, let $\mathcal{K}$ be a $d$-pseudomanifold and $(V(\mathcal{K}), \mathcal{I})$ be a matroid of rank $d+1$ with an ordered basis $B$. Let $\mathcal{K}_{B}$ (resp. $\partial \mathcal{K}_{B}$ ) be the collection of all those $d$-simplexes $\sigma=$ $\left\{v_{0}, v_{1}, \ldots, v_{d}\right\}$ (resp. boundary $(d-1)$-simplexes) of $\mathcal{K}$ such that $\left(v_{0}, v_{1}, \ldots, v_{d}\right)$ (resp. $\left.\left(v_{0}, v_{1}, \ldots, v_{d-1}\right)\right)$ is a $B$-sequence. When $\mathcal{K}$ is orientable with an coherent orientation-valued function $\omega$, let $\mathcal{K}_{B}^{+}$(resp. $\mathcal{K}_{B}^{-}$) be the collection of all those $d$ simplexes $\sigma=\left\{v_{0}, v_{1}, \ldots, v_{d}\right\}$ of $\mathcal{K}$ such that $\left(v_{0}, v_{1}, \ldots, v_{d}\right)$ is a $B$-sequence and $\omega(\sigma)=(+1)\left[v_{0}, v_{1}, \ldots, v_{d}\right]\left(\right.$ resp. $\left.(-1)\left[v_{0}, v_{1}, \ldots, v_{d}\right]\right)$ and let $\partial \mathcal{K}_{B}^{+}\left(\right.$resp. $\left.\partial \mathcal{K}_{B}^{-}\right)$ be the collection of all those boundary $(d-1)$-simplexes $\tau=\left\{v_{0}, v_{1}, \ldots, v_{d-1}\right\}$ of $\mathcal{K}$ such that $\left(v_{0}, v_{1}, \ldots, v_{d-1}\right)$ is a $B$-sequence and the induced orientation on $\tau$ from the orientation $\omega(\sigma)$ on the unique $d$-simplex $\sigma$ of $\mathcal{K}$ having $\tau$ as a $(d-1)$-face is $(+1)\left[v_{0}, v_{1}, \ldots, v_{d-1}\right]$ (resp. $\left.(-1)\left[v_{0}, v_{1}, \ldots, v_{d-1}\right]\right)$. Then, by Theorem 3 with the explanation above, the following Theorem 5 is true.

Theorem 5. Let $\mathcal{K}$ be a d-pseudomanifold and $(V(\mathcal{K}), \mathcal{I})$ be a matroid of rank $d+1$. Then for each ordered basis $B$ of $(V(\mathcal{K}), \mathcal{I})$,

$$
\left|\mathcal{K}_{B}\right| \equiv\left|\partial \mathcal{K}_{B}\right| \quad(\bmod 2)
$$

Suppose further, $(\mathcal{K}, \omega)$ is coherently oriented, then

$$
(-1)^{d}\left\{\left|\mathcal{K}_{B}^{+}\right|-\left|\mathcal{K}_{B}^{-}\right|\right\}=\left|\partial \mathcal{K}_{B}^{+}\right|-\left|\partial \mathcal{K}_{B}^{-}\right| .
$$

An analogous discussion about the special case of Theorem 4 is the notion of Sperner Matroid, in which the matroid dependence and the affine dependence are compatible in a triangulation of a simplex as stated in Theorem 6 .

Theorem 6. Let $T$ be a triangulation of a d-simplex $\overline{a_{0} a_{1} \ldots a_{d}}$ and $(V(T), \mathcal{I})$ be a Sperner matroid over $T$, that is, for each $v \in V(T)$,

$$
v \in \operatorname{conv}\left(\left\{a_{k_{0}}, a_{k_{1}}, \ldots, a_{k_{r}}\right\}\right) \quad \text { implies } \quad v \in \operatorname{cl}\left(\left\{a_{k_{0}}, a_{k_{1}}, \ldots, a_{k_{r}}\right\}\right)
$$

whenever $0 \leq r \leq d$ and $0 \leq k_{0}<k_{1} \ldots<k_{r} \leq d$. If $B=\left(a_{0}, a_{1}, \ldots, a_{d}\right)$ is an ordered basis and if the $(d-1)$-simplex $\overline{a_{1} \ldots a_{d}}$ contains no loops of the matroid $(V(T), \mathcal{I})$, then, with the canonical orientation $\omega$,

$$
\left|\widetilde{T}_{B}^{+}\right|-\left|\widetilde{T}_{B}^{-}\right|=1
$$


where $\widetilde{T}$ is the vertex scheme of $T$.

Theorem 6 was proved by Lee and Shih [7]. We conclude by remarking that the hypothesis " $\overline{a_{1} \ldots a_{d}}$ contains no loops" in Theorem 6 implies the corresponding labelling is Sperner, hence Theorem 6 holds.

\section{REFERENCES}

1. R. B. Bapat, A constructive proof of a permutation-based generalization of Sperner's lemma, Math. Program., 44 (1989), 113-120.

2. K. Fan, Simplicial maps from an orientable $n$-pseudomanifold into $S^{m}$ with the octahedral triangulation, J. Comb. Theory, 2 (1967), 588-602.

3. D. Gale, Equilibrium in a discrete exchange economy with money, Internat, J. Game Theory, 13 (1984), 61-64.

4. B. Knaster, C. Kuratowski, S. Mazurkiewicz, Ein Beweis des Fixpunktsatzes für n-dimensionale Simplexe, Fund. Math., 14 (1929), 132-137.

5. H. W. Kuhn, A new proof of the fundamental theorem of algebra, Math. Program. Study, 1 (1974), 148-158.

6. S. N. Lee and M. H. Shih, A counting lemma and multiple combinatorial Stokes' theorem, European J. Combin., 19 (1998), 969-979.

7. S. N. Lee and M. H. Shih, Sperner matroid, Arch. Math., 81 (2003), 103-112.

8. S. N. Lee and M. H. Shih, Retrieve a Sperner map from a Sperner matroid, Taiwanese J. Math., 10(1) (2006), 181-185.

9. L. Lovász, Matroids and Sperner's lemma, European J. Combin., 1 (1980), 65-66.

10. F. Meunier, Combinatorial Stokes' formulae, European J. Combin., 29 (2008), 286297.

11. J. G. Oxley, Matroid theory, Oxford University Press, New York, 1992.

12. H. Scarf, The approximation of fixed points of continuous mapping, SIAM J. Appl. Math., 15 (1967), 1328-1343.

13. L. S. Shapley, On balanced games without side payments. In: T. C. Hu, M. Robinson, (eds.), Mathematical Program. Math. Res. Cent. Publ., (New York: Academic Press), 30 (1973), 261-290.

14. M. H. Shih and S. N. Lee, A combinatorial Lefschetz fixed-point formula, J. Combin. Theory Ser. A, 61 (1992), 123-129.

15. M. H. Shih and S. N. Lee, Combinatorial formulae for multiple set-valued labellings, Math. Ann., 296 (1993), 35-61.

16. E. Sperner, Neuer Beweis für die Invarianz der Dimensionzahl und des Gebietes, Abh. Math. Sem. Univ. Hamburg, 6 (1928), 265-272. 
17. A. W. Tucker, Some topological properties of disk and sphere, in: Proc. of the First Canadian Mathematical Congress, Montreal 1945, 285-309.

\section{Chien-Hung Chen}

Department of Business Administration,

Transworld Institute of Technology,

Yunlin 640, Taiwan.

E-mail: hung@tit.edu.tw

\section{Shyh-Nan Lee}

Department of Applied Mathematics,

Chung Yuan Christian University,

Chung Li 320, Taiwan.

E-mail: nan@math.cycu.edu.tw

\section{Mau-Hsiang Shih}

Department of Mathematics,

National Taiwan Normal University,

Taipei 116, Taiwan.

E-mail: mhshih@math.ntnu.edu.tw 